\title{
Ignite the Spark of Wisdom_- Thinking on the Cultivation of Elementary Students' Mathematical Intuition Thinking Ability
}

\author{
Jianhua Shen ${ }^{*}$ \\ Ding Hui Experimental Primary School Affiliated to Zhejiang Normal University, Hangzhou, Zhejiang, 310021, China
}

\section{ARTICLE INFO}

Article history

Received: 30 September 2020

Revised: 6 October 2020

Accepted: 9 October 2020

Published Online: 16 October 2020

Keywords:

Cultivation

Intuitive thinking

Primary mathematics

\begin{abstract}
In the learning process of primary mathematics, intuitive thinking remains an important section for students to analyze and solve mathematical problems, which has played an irreplaceable role in enlightening and developing the underlying intellectual and non-intellectual factors of students. By analyzing and comparing the relevant theories and research results regarding mathematical intuition thinking, as well as taking into account the learning characteristics of elementary students, the author has summarized four kinds of strategies suitable for training the mathematical intuition thinking ability of elementary students.
\end{abstract}

\section{Introduction}

$\mathrm{T}$ There is a calculation problem in Sun Zi Suan Jing (Master Sun's Arithmetical Manual), "There are some items, while I don't know the concrete number. If I count them three by three, two will be left; if I count them five by five, three left; and if I count them seven by seven, two left, so what is the quantity of these items?" The famous mathematician Hua Luogeng quickly came up with the answer when he saw the problem for the first time. He thought it this way: if I count them three by three, two left, and if I count them seven by seven, also two left, so the number could be $3 \times 7+2=23$, then divide 5 into 23 , and the answer shall be 3 . Obviously, it is not a general way of thinking, but the intuitive thinking used for solving problems.

Bruner put it that, "Intuition is equal to clever specula- tion, rich hypothesis and experimental conclusions made in a bold and quick manner." ${ }^{[1]}$ Mathematics intuition is a sort of rapid and direct insight or comprehension of a mathematical object by human brains, whose basic form is related to the inspiration and insight of intuition. In the teaching process of primary mathematics, the training of intuitive thinking ability is of great significance to the development of students' thinking flexibility, agility and creativity.

\section{Encourage Conjecture to Develop the Intuitive Thinking Ability}

"To be an excellent mathematician, you must try to be a good guesser in the first place," declared the famous mathematician George Polya ${ }^{[2]}$. In the teaching process, teachers should consciously guide students to make rea-

*Corresponding Author:

Jianhua Shen,

No. 851, Huahe Street, Dinglan Street, Jianggan District, Hangzhou City, Zhejiang Province, China (Dinghui Experimental Primary School Affiliated to Zhejiang Normal University);

E-mail:3214836571@qq.com. 
sonable conjecture, which can be helpful to develop students' intuitive thinking ability.

For example, in the practice lesson of addition and subtraction of fractions, I asked students to make a group of oral arithmetic exercises: $\frac{1}{2}-\frac{1}{7}, \frac{1}{4}+\frac{1}{7}, \frac{1}{3}-\frac{1}{8}$. After checking the answers, one of the students raised his hand and said: "I've found a rule that if you add a fraction by a fraction, the denominator of the sum is the product of the denominator of these two fractions, and the numerator is the sum of these two denominators; In subtraction, the denominator of difference is the product of these two denominators, and the numerator is the difference between them." Clearly, this conjecture was wrong, while I still praised him, then I asked everyone to do a couple of arithmetic problems by using the law of conjecture: $\frac{1}{4}-\frac{1}{10}, \frac{1}{6}$ $+\frac{1}{8}$, soon I found that the conclusion made just now was wrong. Therefore, I asked in time: "Boys and girls, please think it carefully, why is it wrong?" After discussion, it was finally concluded that, "The previous rule should be based on a premise that the denominator of these two fractions shall be a co-prime number." Teachers should affirm students' conjecture in time to make them feel psychologically safe and free, so that they will be bold enough to think, express and guess, and to guess the conclusion of problems and the way of solving them through using reasonable methods of conjecture, as well as being able to guess the possibility from special to general scope and the organic connection between knowledge, thus truly "touching" their own research objects.

\section{Combination of Numbers and Shapes to Cultivate Intuitive Thinking Habit}

Mathematical image intuition remains one of the sources of intuitive thinking in mathematics. In mathematics teaching, we should guide students to make shapes and numbers well-combined through deep observation and association. The intuition induced by virtue of graphic features is of great benefit to cultivate the agility and accuracy of intuitive thinking ${ }^{[3]}$.

For instance, to calculate $\frac{1}{2}+\frac{1}{4}+\frac{1}{8}+\frac{1}{16}+\frac{1}{32}+\frac{1}{64}$ $+\frac{1}{128}$, it can be drawn from the structural feature of the formula that the latter number is always the half of the former one. Figure 1 can be constructed to solve this problem, through which it can be seen that the original formula is equal to $1-\frac{1}{128}=\frac{127}{128}$.

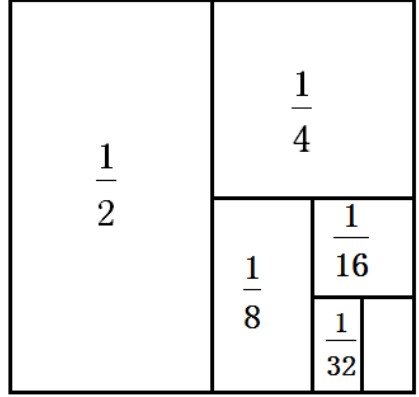

Figure 1

The combination of numbers and shapes is helpful to further understand mathematical problems and enrich students' imagination. Teachers should consciously provide the artistic conception and opportunity of intuitive thinking, dig into a fine example of the combination of numbers and shapes, and make a sound prompt and transformation of the relationship between "number" and "shape", so as to guide students to study problems intuitively by using graphics, and to induce students' intuition by virtue of graphic features. All this will help develop the agility and accuracy of intuitive thinking of students.

\section{Pursue Aesthetic Feelings and Seek for the Source of Intuitive Thinking}

Jacques Solomon Hadamard, a French mathematician, believed that the nature of mathematical intuition is a kind of "sense of beauty" or "aesthetic feeling". Aesthetic feeling of mathematics is a rich source produced by intuition. In mathematics teaching, students should be guided to experience and appreciate the inner beauty of mathematics and cultivate aesthetic consciousness, which also plays an essential part in enhancing their intuition ability ${ }^{[4]}$.

For example, how many cubic centimeters are needed to make the following work piece (Figure 2)?

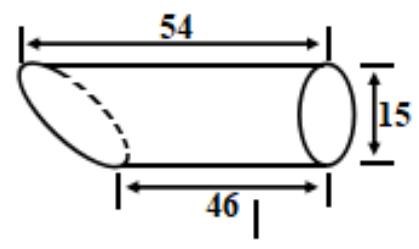

(unit of measurement:CM)

Figure 2

Students are usually dealing with problems of calculating the volume of a regular cylinder, and it seems that they now have no way to start with this problem. While if we tend to think from the perspective of invigorating beauty and turn the irregular into the regular one (Figure 3 ), we can make two identical work pieces into a regular

DOI: https://doi.org/10.26549/jetm.v4i2.5443 
cylinder, and then calculate its volume and divide it by 2 .

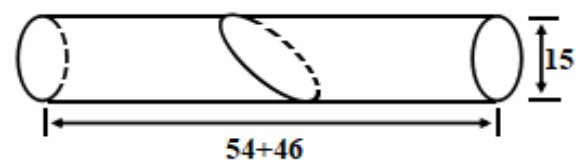

Figure 3

There are abundant aesthetic factors in mathematics itself featuring simplicity, symmetry, similarity, harmony and singularity, which have remained the source of arousing students' interest in learning mathematics, as well as the impetus leading to mathematics intuition. Hence, in teaching practice, teachers can try to inspire students with the beauty of mathematics and guide them to imagine and think by using the principle of beauty, as well as encouraging them to pursue the mathematics beauty, so as to help students realize the transformation from emotional pleasure to rational understanding.

\section{Lay a Solid Foundation and Create Conditions for Intuitive Thinking}

Knowledge and experience remains a precondition for intuitive thinking. Although the acquisition of intuition is accidental, it is definitely not a fantasy, but based on the solid knowledge and experience.

For instance, when calculating $3.625 \times 9.24 \times 0.75 \div\left(9 \frac{6}{25}\right.$ $\times 3 \frac{5}{8} \times \frac{1}{4}$ ), it will be complicated if you do that step-bystep. While if you can see the relationship of the three sets of factors between the dividend and the divisor, namely, 3.625 equals $3 \frac{5}{8}, 9.24$ equals $9 \frac{6}{25}$, and 0.75 is three times as much as a quarter, then you can see at a glance that the answer is 3. Here, the proficient skills of fraction and decimal inter-conversion and the skillful use of operation laws and properties pose a prerequisite for the formation of intuition.

As Bruner said: "Intuitive thinking is always based on being familiar with the area of knowledge and its structure involved, which makes it possible for thinkers to leap forward, surpass and take shortcuts." Therefore, to train students' intuitive thinking ability, the first and the most important thing is to enable them to grasp knowledge they've learned and enrich their daily life and the learning experience of mathematics. Only in this way can we build a bond with the knowledge we've learned and understand their internal connections and differences, and only by doing this can we see through the nature of things from various of intricate mathematical problems and comprehend by analogy.
We emphasize the essential role of intuitive thinking in cultivating creative ability, but we do not negate logical thinking, on the contrary, attention should be paid to the complementary relationship between logical thinking and intuitive thinking. Each of them has a function: intuition is the tool of invention, and logic is the tool of proof. For mathematics, intuition thinking and logical thinking have formed two wings of the development in mathematics, so both of them are equally important. In mathematics teaching, the training of students' intuitive thinking should be closely combined with logical thinking, as well as making sure that the two kinds of thinking supplement each other, so as to cultivate the innovative thinking of students.

"The truly valuable factor in thinking is intuition", declared by Einstein, whoever spoke highly of intuition. Only by relying on the solid and comprehensive knowledge background and teacher's sensitive eyes to find, capture and make persistent training can intuitive thinkingthe bright light of wisdom--flash peculiar lights.

\section{References}

[1] [FA] Adama, translated by Chen Zhiyin and Xiao Xi'an. Discovery psychology in mathematics [M]. Nanjing: Jiangsu Education Press, 1989.

[2] Fu hailun. Attention should be paid to the cultivation of students' intuitive thinking consciousness in mathematics [J]. Bulletin of mathematics. 1997(04): 7-8

[3] Gong Naiwu. On mathematical intuitive thinking and training $[\mathrm{J}]$. Teaching and management. 2005 (04): 50-51.

[4] Huang Jianhua. Training strategy of mathematical intuition thinking for primary school students [J]. Modern primary and secondary education, 2004(09): 27-29.

[5] Huang Xufang. Let intuitive thinking light up mathematics teaching in middle school $[\mathrm{J}]$. Mathematics teaching communication, 2005(219): 12-15.

[6] Li diansen. Bruner's intuitionistic thinking theory and its teaching significance $[\mathrm{J}]$. Foreign education research. 2003 (01): 14-17.

[7] Liu Dianzhi, Zhang Qinglin. On the psychological mechanism of intuition $[\mathrm{J}]$. Educational research, 1988 (01): 48-51.

[8] Liu Xianghong, Zhang Cuizhen. Pay attention to the cultivation of primary school students' mathematical intuitive thinking ability [J]. Subject teaching exploration. 2005 (04): 44-45.

[9] Ren Zhanghui. Mathematical thinking theory [M]. Nanning: Guangxi Education Press, 2001. [10] Wang 
Chongjiu. Cultivation of primary school students' mathematical intuitive thinking ability [J]. Education and Research Forum, 2008(03): 11-12.

[11] Wang Jiacong. Where does mathematical intuition come from [J]. Middle school mathematics monthly, 2004(10): 3-4.

[12] Xu Lizhi, Xu benshun. Mathematical beauty and aesthetics in Mathematics Teaching [J]. Shandong education, 1997(11):30-35.

[13] Zhao Silin, Wu Libao. Research on the cultivation of mathematical intuitive thinking ability [J]. Teaching and management. 2009(05):130-131.

\section{About the Author}

Jianhua Shen, from Hangzhou, Zhejiang. In 2014, he obtained the senior teacher qualification of middle school. He is currently a master tutor of Hangzhou Normal University and the principal of Dinghui Experimental Primary School affiliated to Zhejiang Normal University. He has been teaching for more than 20 years and has extensive teaching and management experience. He has presided over a number of national, provincial and municipal research projects. He has published more than 10 papers in journals above the provincial level, many of which have won provincial, municipal, and district awards. 